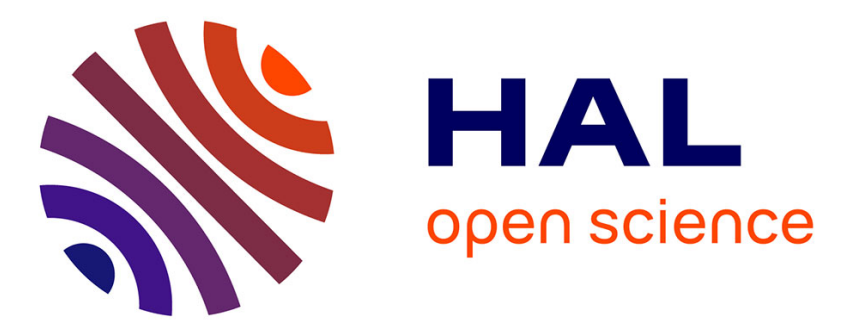

\title{
Short-term dynamics of a density interface following an impact
}

\author{
Arnaud Antkowiak, Nicolas Bremond, Stéphane Le Dizès, Emmanuel \\ Villermaux
}

\section{- To cite this version:}

Arnaud Antkowiak, Nicolas Bremond, Stéphane Le Dizès, Emmanuel Villermaux. Short-term dynamics of a density interface following an impact. Journal of Fluid Mechanics, 2007, 577, pp.241-250. 10.1017/S0022112007005058 . hal-00126025

\section{HAL Id: hal-00126025 \\ https://hal.science/hal-00126025}

Submitted on 24 Jan 2007

HAL is a multi-disciplinary open access archive for the deposit and dissemination of scientific research documents, whether they are published or not. The documents may come from teaching and research institutions in France or abroad, or from public or private research centers.
L'archive ouverte pluridisciplinaire HAL, est destinée au dépôt et à la diffusion de documents scientifiques de niveau recherche, publiés ou non, émanant des établissements d'enseignement et de recherche français ou étrangers, des laboratoires publics ou privés. 


\title{
Short-term dynamics of a density interface following an impact
}

\author{
By A. ANTKOWIAK, N. BREMOND, S. LE DIZÉS \\ AND E. VILLERMAUX†
}

IRPHÉ, Aix-Marseille Université, CNRS, Technopôle de Château-Gombert 49, rue Frédéric Joliot-Curie 13384 Marseille Cedex 13, France.

(Received 20 January 2007)

A tube filled with a perfectly wetting liquid falls axially by its own weight. In its gravity free reference frame, the liquid interface deforms by surface tension into a hemispherical shape. At the impact of the tube on a rigid floor, the interface curvature reverses violently, forming a concentrated jet. If the contact angle at the tube wall is such that the interface is flat, the liquid rebounds as a whole with the tube, with no deformation. We analyze this phenomenon using an impulse pressure description, providing an exact description of the initial liquid velocity field at the impact, supported by high speed image velocimetry measurements. This initial dynamics is insensitive to liquid surface tension and viscosity.

\section{Introduction}

Liquid jets, or ligaments are the sinews of atomization (Villermaux 2007) as drops always result from the rupture of more or less smooth threads. Ubiquitous features of free surface flows, jets naturally erupt in a wide variety of situations, including bursting bubbles (Blanchard 1967), overdriven Faraday waves (Longuet-Higgins 1983) or collapsing voids (Benjamin \& Ellis 1966) as classical examples. Another common occurrence of jet formation is the one resulting from an impact. This situation is illustrated on figure 1 with the paradigmatic experiment referred to as 'Pokrovski's experiment' in Lavrentiev \& Chabat (1980): a glass tube filled with water falls axially by its own weight. In its gravity free reference frame, the liquid interface deforms by surface tension into a closeto-hemispherical shape (meniscus) to adapt to the wetting condition at the wall. At the impact of the tube on a rigid floor, the interface curvature reverses violently, forming a concentrated jet. Interestingly, when the tube wall is altered such that the contact angle is around $90^{\circ}$, the interface is initially flat, and not deformed after rebound (figure 21. This simple experiment singles out the crucial role of the free surface geometry on its later evolution. Such an important effect associated with the geometry of the free surface has been known for more than a century in the context of 'shaped-charge jets' (Birkhoff et al. 1948). Military devices exploiting this effect basically consist in lining an explosive charge with an artificial 'meniscus' made of metal that collapses with the detonation wave and produces an intense metallic liquid jet. Whereas the resulting jet velocity typically scales with the detonation wave celerity (of order of several kilometers per second), the jet produced here with the impacted tube rather scales with the impact velocity, suggesting a different mechanism of jet formation. 


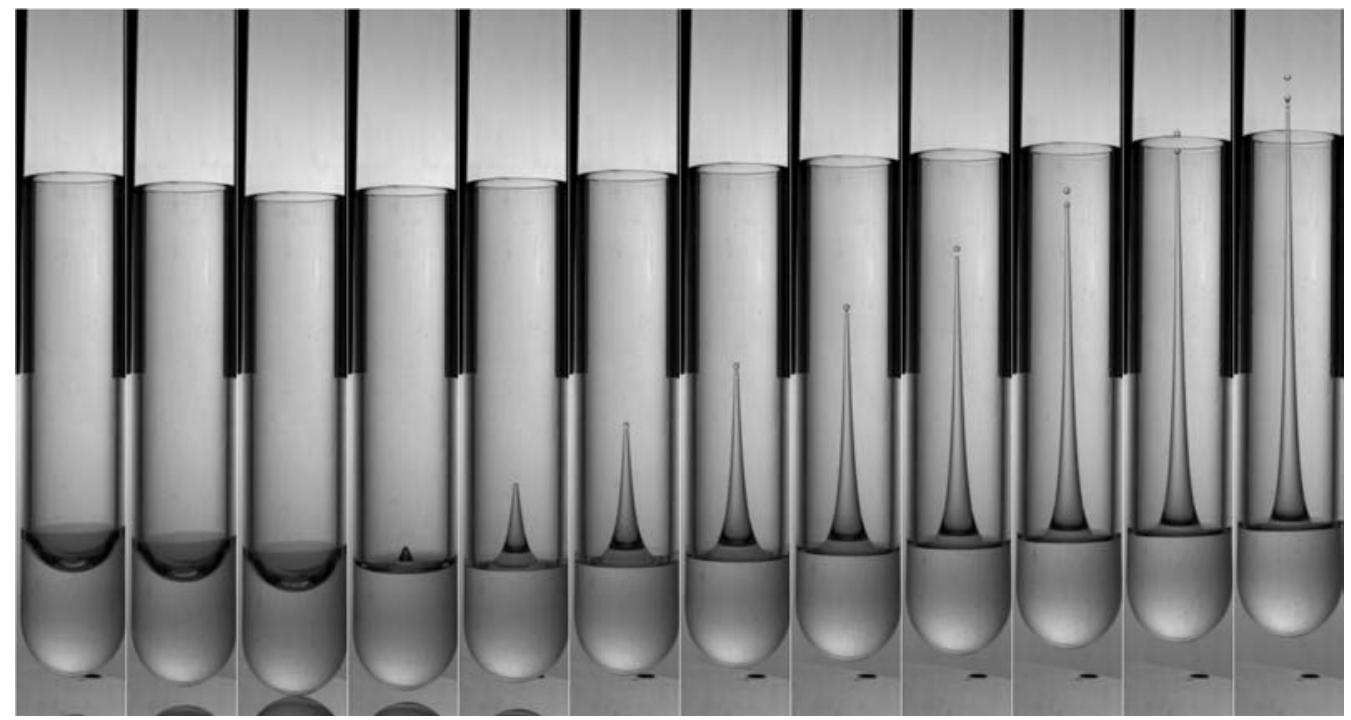

Figure 1. A tube filled with a wetting liquid and falling under gravity gives birth to a strong jet after impact. The interval separating each snapshot of this sequence is $5.5 \mathrm{~ms}$.

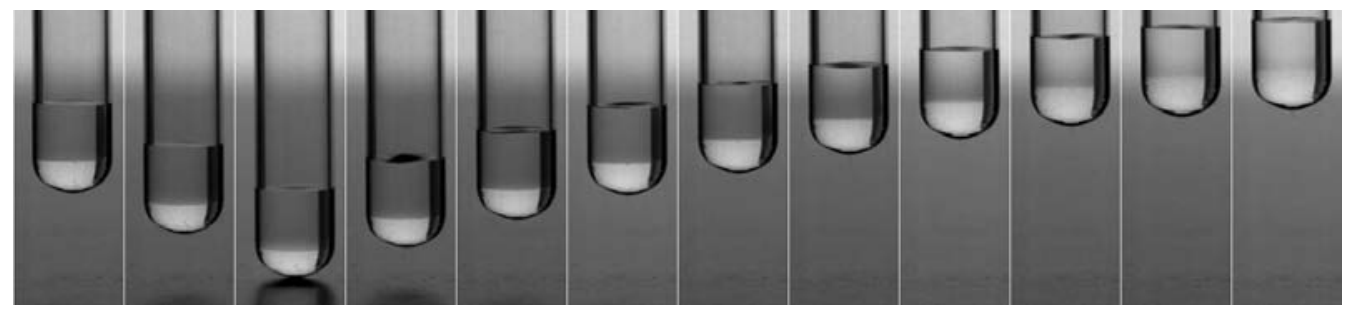

Figure 2. When the tube wall is made hydrophobic (silanization), the interface is initially flat, and not deformed after rebound.

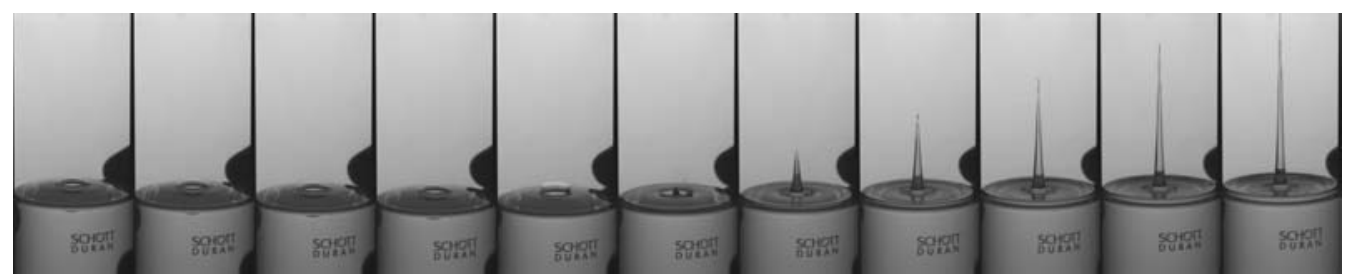

FIgURE 3. A liquid with a free surface is falling as a whole under gravity. A bubble at the free surface gives rise to a strong jet after impact. The interval separating each snapshot of this sequence is $1 \mathrm{~ms}$.

The purpose of the present study is to elucidate the role of free surface corrugations, not only associated with menisci, but also with standing waves or bubbles cavities (see figure 3 on the initial interface dynamics. We proceed as follows: in $\S 2$, employing a pressure impulse approach (Batchelor 1967; Cooker \& Peregrine 1995), the pressure field associated with impact as well as the corresponding velocity field are derived using a variant of the multipole expansion. In $\S 3$, the theoretical results are compared to measurements of the liquid displacement field using high-speed video and particle image velocimetry. The roles of confinement, surface tension (including the case of non cohesive fluids) and viscosity are finally discussed. 
(a)

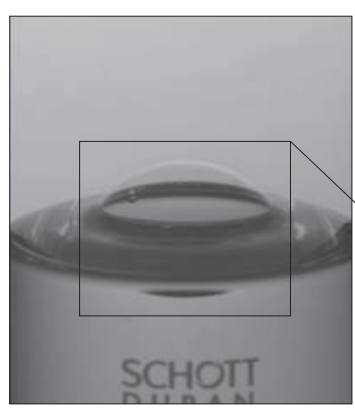

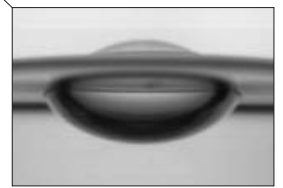

(b)

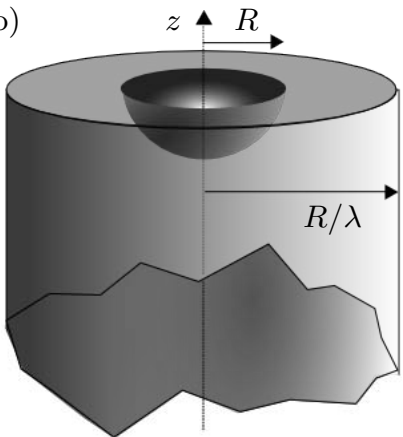

FiguRE 4. Left: Typical experiment of a cavity produced with a bubble, just before impact. Right: sketch of the liquid domain in the theoretical idealization.

\section{Pressure impulse in an impacted tube}

A sudden change in the tube velocity induces pressure gradients which in turn produce a sudden change in the liquid velocity (Batchelor 1967, §6.10). Looking closer at the very short time dynamics, the impact pressure field is established via a complex acoustic field radiated from the boundaries. Typically, these phenomena occur on a few acoustic timescales corresponding to what we call the impact duration of Over the impact period, neither the velocity nor its spatial gradients are expected to balance the dominant time derivative, such that the dynamics is assumed to be governed by:

$$
\frac{\partial \boldsymbol{u}}{\partial t}=-\frac{1}{\rho} \nabla p .
$$

As commonly done in impact studies (Cooker \& Peregrine 1995), the intricate details of the acoustic field are not examined and an incompressible evolution ruled by global momentum balances is considered, just as in the problem of billiard balls impact where the elastic wavefield is classically neglected. On integrating equation (2.1) over the impact duration, the velocity field after impact is uncovered:

$$
\boldsymbol{u}(\tau)-\boldsymbol{u}(0)=-\frac{1}{\rho} \nabla P,
$$

where $P$ is the impulse pressure defined by:

$$
P=\int_{0}^{\tau} p \mathrm{~d} t
$$

accounting for the total liquid pressure variation associated with impact (or impulse gravity). Incompressibility implies that $P$ is an harmonic function:

$$
\Delta P=0 .
$$

Note that the neglected viscous terms are actually identically nil, the velocity field being the gradient of an harmonic potential. Consequently, there is no viscous effects in the volume, and the region of influence of viscosity is concentrated near the free surface and the wall in a thin boundary layer of thickness $\sqrt{\nu \tau}$, with $\nu$ the kinematic viscosity.

The purpose of the following developments is to derive the solution of the Laplace equation (2.4) for the physical setup sketched in figure 4. It consists of an infinite

$\dagger$ Here, $\tau$ is observed to be $10^{-4} s$, whereas the acoustic timescale is rather $10^{-5} s$. 
vertical tube of radius $R / \lambda$ filled with liquid. At the free surface, a bubble of radius $R$ $(\leq R / \lambda$, so that the 'confinement factor' $\lambda$ lies in the range $0<\lambda \leq 1)$, modelled with an half-sphere, is present. At initial time, the container is impulsively started, with a velocity $U_{0} \boldsymbol{e}_{\boldsymbol{z}}$. The associated impulse pressure satisfies $P=0$ at the free surface. On the solid boundaries, the boundary conditions derive from 2.2 :

$$
U_{n}=-\frac{1}{\rho} \frac{\partial P}{\partial n},
$$

where $U_{n}=U_{0}\left(\boldsymbol{e}_{\boldsymbol{z}} \cdot \boldsymbol{n}\right)$. Moreover, at the infinitely deep bottom, the impact enforces $\frac{\partial P}{\partial z}=-\rho U_{0}$.

Were no bubble present, the trivial solution $-\rho U_{0} z$ would hold for the impulse pressure field. This 'impulsive hydrostatic' pressure distribution imparts an equal momentum distribution within the liquid bulk. Each fluid particle acquires a velocity $U_{0} \boldsymbol{e}_{\boldsymbol{z}}$ after impact in the fixed frame, that is no velocity at all in the moving frame: incompressibility guarantees that the sudden change in the container velocity is instantaneously transmitted to all fluid particles through the pressure linear stratification. This instructive example, illustrated with the experiment showed figure 2, shows that the only free surface geometry compatible with the preceding impulsive hydrostatic pressure distribution is a planar one. Indeed, enforcing a zero pressure distribution on a curved surface will generate corrections to the pressure field. This situation is the one under study: free surface corrugations, possibly produced with the help of surface tension, but not exclusively, will give rise to pressure and velocity corrections.

Analytically, solutions of Laplace equation are easily found when the boundaries of the domain are iso-coordinates surfaces. Conversely, the present problem is 'hybrid' in this sense as the boundaries are iso-coordinates of two representation systems. Seventy years ago, Knight (1936) made a first step in building solutions to such 'mixed' problems, considering precisely a problem with an identical geometry. Closely following the original treatment of Knight, we shall now investigate and solve the pressure impulse problem.

\subsection{Elementary harmonic solutions in the cylindro-spherical domain: modified multipoles}

In the following, each considered field will admit two representations : a cylindrical one $(\xi, \Phi, z)$ and a spherical one $(r, \theta, \Phi)$. With these two alternate views, it is possible to express the requirements on the general solution $F$ to the present problem:

$$
\left\{\begin{array}{l}
\text { (i) } \Delta F=0 \text { in the whole domain }(r \geq R, \xi \leq R / \lambda, z \leq 0) \\
\text { (ii) } \frac{\partial F}{\partial \xi}=0 \text { on the cylinder surface } \xi=R / \lambda \\
\text { (iii) } F=0 \text { on the plane } z=0 \\
\text { (iv) } F=0 \text { on the sphere surface } r=R
\end{array}\right.
$$

Without condition (ii), expressing the impermeability condition on the cylinder, a standard approach would consist in an expansion of the solution into odd multipoles (each readily satisfying (i) and (iii)) so as to meet condition (iv) on the sphere. With the cylinder included, the strategy basically remains similar, but the base functions are modified with the help of regular cylindrical harmonic functions in order to satisfy (ii).

\subsubsection{The modified dipole}

The dipolar field $\phi(r, \theta)=-\frac{\partial}{\partial z}\left(\frac{1}{r}\right)=\cos \theta / r^{2}$, which is a singular solution to Laplace equation in spherical coordinates, fails to meet condition (ii) as already noticed. It is 
therefore desirable to build a solution of the form $F_{0}=\phi(r, \theta)+\varphi(\xi, z)$, where $\varphi(\xi, z)$ is a regular solution to Laplace equation in cylindrical coordinates preserving $(i)$ and (iii) but derived so that $F_{0}$ complies with (ii). Regularity and symmetry requirements suggest the following form for $\varphi$ :

$$
\varphi(\xi, z)=\int_{0}^{\infty} f(m) \sin (m z) I_{0}(m \xi) \mathrm{d} m
$$

where the spectral decomposition $f(m)$ is unknown for the moment. On the cylinder surface, the derivatives with respect to $\xi$ of the regular and singular parts must balance each other:

$$
-\frac{\partial \phi}{\partial \xi}(R / \lambda, z)=3 \frac{\left(\frac{R}{\lambda}\right) z}{\left(\left(\frac{R}{\lambda}\right)^{2}+z^{2}\right)^{5 / 2}}=\frac{\partial \varphi}{\partial \xi}(R / \lambda, z)=\int_{0}^{\infty} m f(m) \sin (m z) I_{1}(m R / \lambda) \mathrm{d} m
$$

Inverting the sine Fourier transform then provides with the following expression for the spectral amplitude:

$$
f(m)=\frac{2}{\pi} \int_{-\infty}^{0} \frac{\sin (m z)}{m I_{1}(m R / \lambda)}\left\{3 \frac{\left(\frac{R}{\lambda}\right) z}{\left(\left(\frac{R}{\lambda}\right)^{2}+z^{2}\right)^{5 / 2}}\right\} \mathrm{d} z .
$$

This spectral decomposition allows to recover the expression of $\varphi(\xi, z)$ :

$$
\varphi(\xi, z)=\frac{2}{\pi} \int_{0}^{\infty} m \frac{K_{1}(m R / \lambda)}{I_{1}(m R / \lambda)} \sin (m z) I_{0}(m \xi) \mathrm{d} m,
$$

where the following relation has been used:

$$
\int_{-\infty}^{0} \frac{a z \sin (m z)}{\left(a^{2}+z^{2}\right)^{5 / 2}} \mathrm{~d} z=\frac{1}{3} m^{2} K_{1}(m a) .
$$

Relation 2.10 explicits the correction to the dipolar field needed so that the resulting modified dipole $F_{0}$ satisfies (i), (ii) and (iii).

Although exact, relation 2.10 does not appear to have the most relevant form in view of the formulation of condition (iv) on the sphere. A more appropriate form of the solution in the vicinity of the sphere consists in a zonal harmonics expansion. On making use of the following identity (Knight 1936):

$$
\sin (m z) I_{0}(m \xi)=\sum_{n=0}^{\infty}(-1)^{n} \frac{m^{2 n+1} r^{2 n+1}}{(2 n+1) !} P_{2 n+1}(\cos \theta)
$$

where $P_{2 n+1}(\cos \theta)$ is the Legendre polynomial of degree $2 n+1$, one can also obtain the following expression for the modified dipole:

$$
F_{0}(r, \theta)=\left(\frac{R}{r}\right)^{2} \cos \theta+\sum_{n=0}^{\infty} \lambda^{2 n+3} \alpha_{2 n+1}^{(0)}\left(\frac{r}{R}\right)^{2 n+1} P_{2 n+1}(\cos \theta),
$$

with

$$
\alpha_{2 n+1}^{(0)}=\frac{(-1)^{n}}{(2 n+1) !} \frac{2}{\pi} \int_{0}^{\infty} m^{2 n+2} \frac{K_{1}(m)}{I_{1}(m)} \mathrm{d} m .
$$

\subsubsection{Modified multipoles}

As remarked by Knight, differentiating $F_{0}$ with respect to $z$ an even number of times gives functions sharing analogous properties, namely harmonicity (i), boundary condi- 
tion (ii) as well as condition (iii). Upon recalling that:

$$
\begin{gathered}
-\frac{\partial^{2 s+1}}{\partial z^{2 s+1}}\left(\frac{1}{r}\right)=\frac{\partial^{2 s}}{\partial z^{2 s}}\left(\frac{\cos \theta}{r^{2}}\right)=(2 s+1) ! \frac{P_{2 s+1}(\cos \theta)}{r^{2 s+2}}, \\
\frac{1}{(2 s) !} \frac{\partial^{2 s}}{\partial z^{2 s}} \sum_{n=0}^{\infty} r^{2 n+1} P_{2 n+1}(\cos \theta)=\sum_{n=0}^{\infty} C_{2 n+2 s+1}^{2 s} r^{2 n+1} P_{2 n+1}(\cos \theta),
\end{gathered}
$$

these solutions can be written as:

$$
\begin{aligned}
F_{2 s} & =\frac{R^{2 s}}{(2 s) !} \frac{\partial^{2 s} F_{0}}{\partial z^{2 s}} \\
& =(2 s+1)\left(\frac{R}{r}\right)^{2 s+2} P_{2 s+1}(\cos \theta)+\sum_{n=0}^{\infty} \lambda^{2 n+2 s+3} \alpha_{2 n+1}^{(2 s)}\left(\frac{r}{R}\right)^{2 n+1} P_{2 n+1}(\cos \theta)
\end{aligned}
$$

where:

$$
\alpha_{2 n+1}^{(2 s)}=C_{2 n+2 s+1}^{2 s} \alpha_{2 n+2 s+1}^{(0)} .
$$

\subsection{Formal expansion of the complete solution}

Having derived the modified multipoles, it is natural to seek the complete solution under an expansion of the form $P=\sum_{0}^{\infty} A_{2 s} F_{2 s}$. However, since the functions $F_{2 s}$ are expected to decrease asymptotically to 0 for large $z$ as $F_{0}$, it appears difficult to comply with the additional constraint $\lim _{z \rightarrow-\infty} \frac{\partial P}{\partial z}=-\rho U_{0}$. While the suggested expansion is natural in the context of a decaying electrostatic potential field created with a charged sphere (the original context of Knight), it fails here to capture the solution. Indeed, elementary solutions of Laplace equation linear in cartesian variables cannot be expressed as a convergent serie of modified multipoles and have therefore to be included separately. More specifically, the 'impulsional hydrostatic' pressure field $-\rho U_{0} z$ already evoked earlier has here to be added to the complete solution:

$$
P(r, \theta)=-\left(\frac{r}{R}\right) P_{1}(\cos \theta)+\sum_{s=0}^{\infty} A_{2 s} F_{2 s},
$$

with $P$ here expressed in units of $\rho U_{0} R$.

In the case of a charged sphere, the potential is forced through inhomogeneous boundary conditions on the sphere surface. Conversely, the impact induces a linear pressure profile, uncompatible with pressure cancellation on the (non-planar) free surface. The impact therefore drives in return the emergence of a multipolar field.

Having included this forcing, the linear relationship between the coefficients of the expansion derives from the constraint of vanishing pressure on the bubble surface. Once projected on each Legendre polynomial $P_{2 n+1}(\cos \theta)$, this reads:

$$
(2 n+1) A_{2 n}+\sum_{s=0}^{\infty} \lambda^{2 n+2 s+3} \alpha_{2 n+1}^{(2 s)} A_{2 s}=\delta_{0 n} .
$$

In the case of weak confinement (e.g. isolated bubble), the first order approximation of these coefficients is:

$$
\left\{\begin{array}{rl}
A_{0} & =1-\lambda^{3} \alpha_{1}^{(0)} \\
A_{2 n} & =-\lambda^{2 n+3} \alpha_{2 n+1}^{(0)} /(2 n+1)
\end{array},\right.
$$

revealing the dominance of the dipolar field in the correction for a large range of confinements. But in the general case where confinement cannot be introduced perturbatively, 
(a)

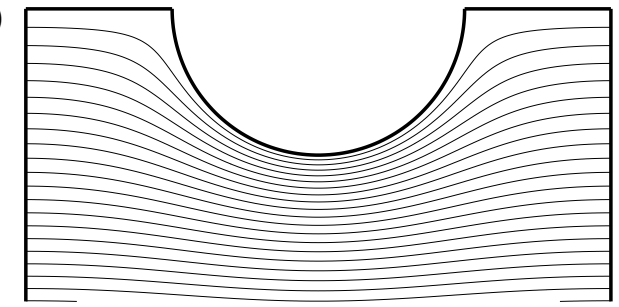

(c)

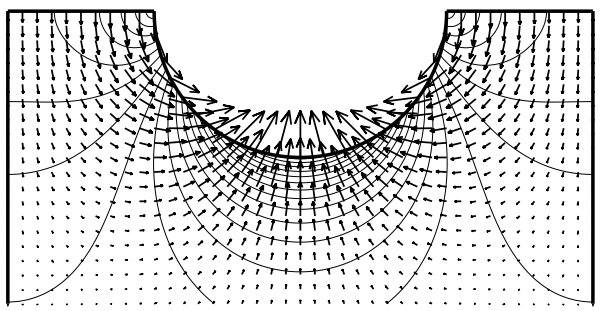

(b)

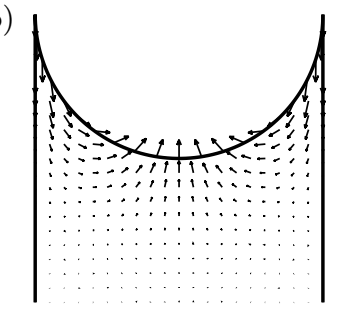

(d)

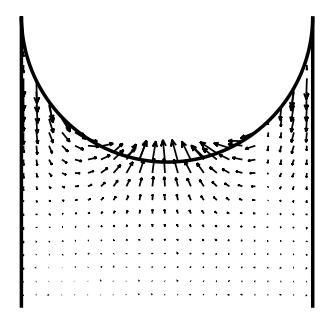

FIGURE 5. Left: pressure levels (a) and velocity field (c) obtained with the cylindrical representation for a value of the confinement factor of $\lambda=0.5$. (a) : the isobars are equispaced with a step of $0.08 \rho U_{0} R$. (c) : along with the velocity vectors are represented the levels of the pressure gradient norm. These are equispaced with a step of $0.12 \rho U_{0}$. Right: the theoretical velocity field for the confined geometry $(\lambda=1)$ is represented figure (b), whereas a typical velocity field obtained experimentally after PIV treatment is plotted figure (d).

the solution of the system 2.19 can still be obtained by numerical means, after truncation at a satisfying order. In all cases, inclusion of 10 modified multipoles appears sufficient to guarantee the respect of condition (iv) with an absolute error inferior than $10^{-4}$.

Eventually, given the general expression for the impulse pressure 2.18 derived, and with the help of the governing equation (2.1), the liquid velocity field in a frame moving with the container reads:

$$
\boldsymbol{u}(r, \theta)=-U_{0} R \boldsymbol{\nabla}\left(\sum_{s=0}^{\infty} A_{2 s} F_{2 s}\right) .
$$

Figure 5 illustrates typical pressure impulse distributions for different confinement factors. The strong inhomogeneity of the pressure gradient field is remarkable and entirely induced by the corrugation of the free surface (driving the emergence of multipoles through condition $(i v)$ ). Immediately after impact, the strongly inhomogeneous pressure field induced by the impact is converted into a similarly inhomogeneous kinetic energy distribution, through the governing equation (2.1). The corresponding velocity fields are represented figure $5 \mathrm{~b}-\mathrm{c}$.

From the above analysis, the following physical picture emerges: introducing a free surface corrugation of, say, depth $H$ and typical curvature radius $R$ induces a deformation of the isobars surrounding the cavity, as illustrated figure 5 . The isobar squeeze then naturally reflects in the pressure gradient intensity in the vicinity of the cavity bottom. Taking the pressure level at the cavity depth far from the corrugation, i.e. $\rho U_{0} H$, and the natural length scale of the cavity, i.e. $R$, as characteristic scales, the order of magnitude of the pressure gradient is expected to be

$$
\frac{\partial P}{\partial z} \approx-\rho U_{0} \frac{H}{R}
$$



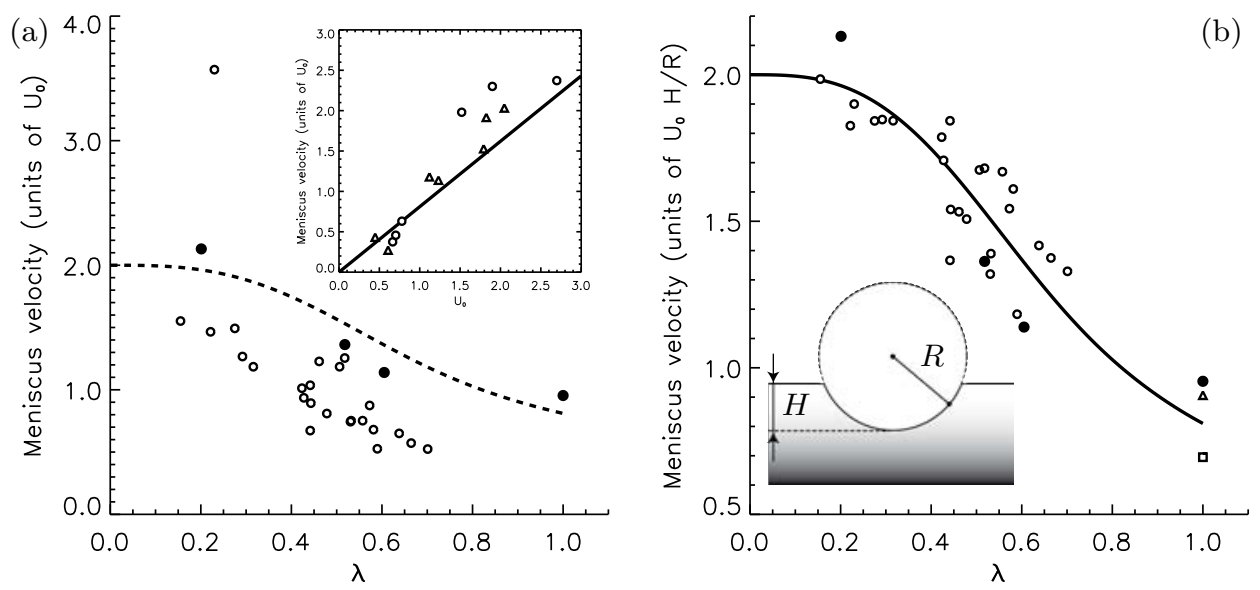

FiguRE 6. Left: maximum meniscus velocity at the impact in a frame moving with the container in units of $U_{0}$, as a function of the confinement factor $\lambda$. The dotted line corresponds to the theoretical prediction, and the circles to the experimental observation. The plain circles correspond to near hemispherical bubbles $(H / R \sim 1)$. The inserted plot illustrates the linear dependence between the meniscus velocity and the impact velocity in the confined $(\lambda=1)$ case for two tubes (circles: glass bottom; triangles: aluminium bottom). Right: Same data as in the left figure, but rescaled with the aspect ratio $H / R$ of the bubble. Water is the working fluid; the triangle reports an experiment made with ethanol (of surface tension 3 times smaller than water), and the square an experiment conducted with the V50 silicon oil of dynamic viscosity 50 times larger than water.

Although $H$ and $R$ are basically the same quantity in the theoretical idealization, this scale distinction will prove useful in the analysis of the experimental results.

\section{Experiments and comparisons}

Experiments have been conducted using a high-speed Photron video camera at typically 3000 frames per second at a resolution of $1024 \times 1024$ pixels. Theory predicts a decrease of the initial meniscus maximal velocity (on the tube axis) as the confinement factor $\lambda$ tends to 1 . This behaviour, already visible from a comparison of the velocity fields plotted in figures 50 and 5, is more detailed in figure 6. In a frame moving with the container, the initial velocity at the cavity bottom has been reported for different confinement ratios and compared with the theoretical prediction. Interestingly, the agreement is correct only for near hemispherical bubbles having comparable curvature radius and cavity depth. Actually, our theory does not consider cavities such that $H / R \neq 1$ (with $H$ and $R$ defined figure 6b). But so far, the mechanism described below equation (2.21) anticipates a linear dependence of the cavity velocity with $H / R$. And indeed, dividing all the observed velocities with this geometrical quantity leads to a quite nicer agreement between theory and experiments (figure 6b).

The structure and intensity of the velocity field was measured using PIV at the moment of impact in the confined geometry $(\lambda=1)$ by seeding the liquid (water) with $100 \mu \mathrm{m}$ particles. A vertical plane containing the tube symmetry axis was illuminated with a laser sheet produced with a continuous $2 W$ Argon laser. Classical PIV treatment (Meunier \& Leweke 2003) gives access to the bulk velocity field. Typical result is shown figure $5 \mathrm{~d}$, in qualitative agreement with the theoretical velocity field (figure $5 \mathrm{~b}$ ). Quantitative comparison is provided by examining the radial and axial velocity profiles along a horizontal line tangenting the meniscus, and the tube axis of symmetry as shown 

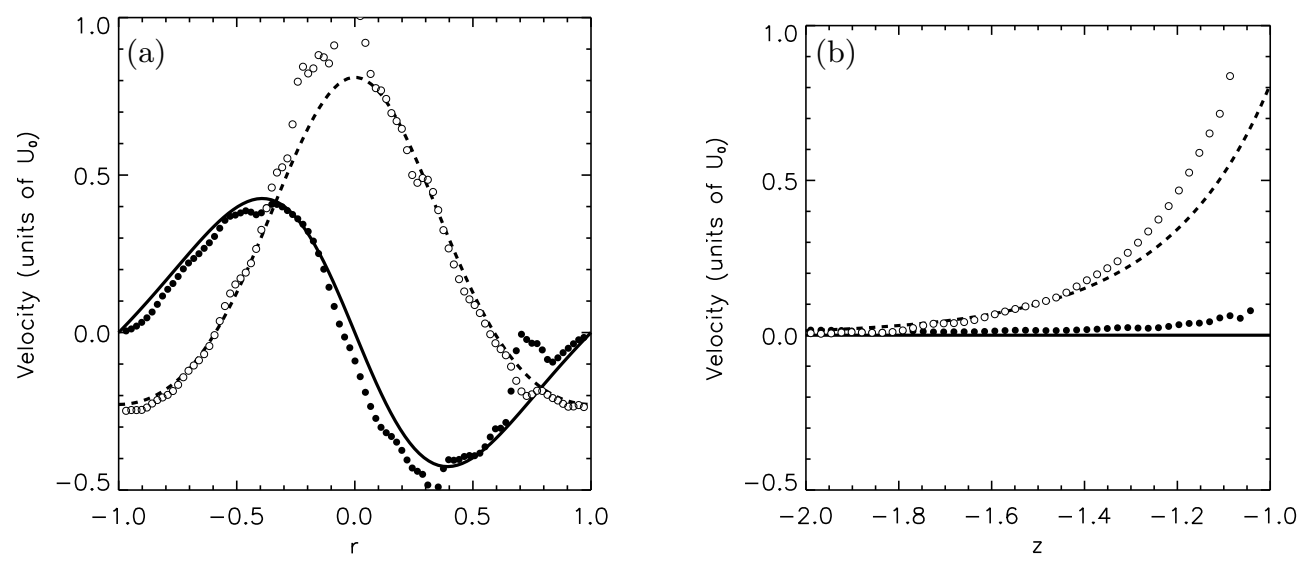

FiguRE 7. Comparison between theoretical and experimental velocity profiles, in units of $U_{0}$ in the confined geometry $(\lambda=1)$. (a): the profile is taken along a ray tangent to the meniscus. (b): the profile is plotted along the symmetry axis. In both cases, the thick (dotted) line correspond to the radial (axial) theoretical velocity. The filled (empty) circles represent an experimental observation made with water, in a $3 \mathrm{~cm}$ diameter glass tube. The impact velocity is $6 \mathrm{~m} / \mathrm{s}$.

on figure 7. This quantitative agreement is achieved within a purely inertial theory accounting for the deformation of the hydrostatic impulse pressure field by the presence of the cavity, disregarding viscous and surface tension stresses. In particular, the viscous boundary layer at the tube wall is invisible (figure $7 \mathrm{a}$ ).

\section{Conclusions and extension to non-cohesive fluids}

The early time deformation of a curved density interface following an impact has been elucidated. An impact induces an impulsional linear pressure stratification within the bulk, therefore transmitting the information of sudden velocity change to all fluid particles. For the fluid to move as a whole, the free surface has to be planar. Any departure from the plane will induce impulse pressure corrections, and hence motions within the bulk. This resulting velocity field has been derived analytically for free surface corrugations ranging from isolated bubbles to menisci by means of a purely inertial theory, in agreement with experimental observations. Viscous effects do not appear to be relevant in the present problem (no visible effect associated with viscous boundary layers nor deviation of the cavity velocity with an oil 50 times more viscous than water), consistently with our description.

Interestingly, the free surface corrugations considered experimentally could not have been produced without the help of surface tension since it is necessary in the formation of menisci, or bubbles. But the dynamics subsequent to the impact does not rely on surface tension at all. A further proof of the non-role played by surface tension is to consider a non-cohesive deformable medium, namely sand as working fluid. A first experiment consists in observing the impact of a tube filled with sand presenting a planar free surface (figure 8). Here, no jet at all is observed and the sand particles are at best superficially fluidized. In a second experiment, an artificial 'meniscus' is sculpted at the free surface and a strong jet is observed in return just after the impact, alike the one observed after the impact of a sphere on sand bed (Thoroddsen \& Shen 2001). The noncohesive character of the medium certainly draws aside surface tension as relevant in the present experiment. Moreover, the fact that a jet emerges in that case too pinpoints 

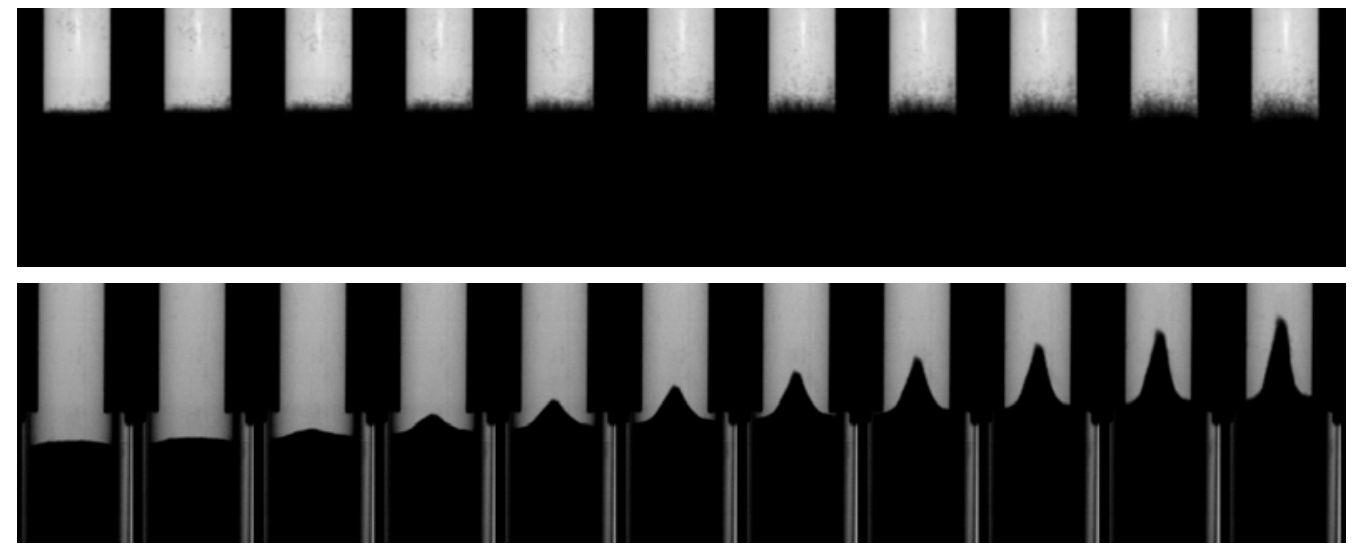

FiguRE 8. Top: a tube filled with sand and with a flat surface is impacted. 2 ms separates each snapshot of the sequence. Bottom: the same tube with a sculpted 'meniscus' is impacted. There is a time interval of $1 \mathrm{~ms}$ between each image.

the universal/ubiquitous aspect of jet formation in a medium with a curved stress-free surface and initial harmonic pressure (or stress) distribution.

The subsequent development of the free surface which rapidly evolves both in shape and velocity is therefore expected to be insensitive to surface tension and viscosity. The features of the resulting stretched jet, the regions and timescales over which viscosity and surface tension will eventually come into play and the ultimate fragmentation of the jet into droplets are currently under investigation.

This work was supported by the Agence Nationale de la Recherche through grant ANR05-BLAN-0222-01, and by EADS Foundation.

\section{REFERENCES}

Batchelor, G. K. 1967 An introduction to fluid dynamics. Cambridge University Press.

Benjamin, T. B. \& Ellis, A. T. 1966 A discussion on deformation of solids by the impact of liquids, and its relation to rain damage in aircraft and missiles, to blade erosion in steam turbines, and to cavitation erosion. Philos. Trans. R. Soc. London Ser. A 260 (1110), $221-240$.

Birkhoff, G., MacDougall, D. P., Pugh, E. M. \& Taylor, G. I. 1948 Explosives with lined cavities. J. Appl. Phys. 19, 563-582.

Blanchard, D. C. 1967 From raindrops to volcanoes. Doubleday, reedited by Dover in 2004.

Cooker, M. J. \& Peregrine, D. H. 1995 Pressure-impulse theory for liquid impact problems. J. Fluid Mech. 297, 193-214.

Knight, R. C. 1936 The potential of a sphere inside an infinite circular cylinder. Quart. J. Math. (Oxford series) 7, 124-133.

Lavrentiev, M. \& Chabat, B. 1980 Effets hydrodynamiques et modèles mathématiques. Éditions MIR, translated from the 1977 russian edition.

Longuet-Higgins, M. S. 1983 Bubbles, breaking waves and hyperbolic jets at a free surface. J. Fluid Mech. 127, 103-121.

Meunier, P. \& Leweke, T. 2003 Analysis and treatments of errors due to high velocity gradients in particle image velocimetry. Exp. Fluids 35, 408-421.

Thoroddsen, S. T. \& Shen, A. Q. 2001 Granular jets. Phys. Fluids 13 (1), 4-6.

Villermaux, E. 2007 Fragmentation. Annu. Rev. Fluid Mech. 39, 419-446. 